\title{
Response of Forced Expiratory Volume/Forced Vital Capacity Ratio to Acapella Device and Breathing Exercises After Upper Abdominal Surgeries
}

\author{
Nesma M. Allam ${ }^{1}$, Mohammed M. Khalaf ${ }^{1}$, Wael N. Thabet ${ }^{2}$, Zizi M. Ibrahim ${ }^{1}$ \\ ${ }^{1}$ Department of Physical Therapy for Surgery, Faculty of Physical Therapy, Cairo University, Cairo, Egypt \\ ${ }^{2}$ Department of general surgery, Faculty of Medicine, Cairo University, Cairo, Egypt
}

Email address:

Dr.nesma2011@yahoo.com (N. M. Allam)

\section{To cite this article:}

Nesma M. Allam, Mohammed M. Khalaf, Wael N. Thabet, Zizi M. Ibrahim. Response of Forced Expiratory Volume/Forced Vital Capacity Ratio to Acapella Device and Breathing Exercises After Upper Abdominal Surgeries. Journal of Surgery. Special Issue: Surgical Infections and Sepsis. Vol. 4, No. 3-1, 2016, pp. 21-24. doi: 10.11648/j.js.s.2016040301.14

Received: February 14, 2016; Accepted: February 15, 2016; Published: March 23, 2016

\begin{abstract}
Background: A major decline in pulmonary function is observed on the first day after upper abdominal surgery. This decline can reduce vital and inspiratory capacity and can culminate in restrictive lung diseases that cause atelectasis, reduced diaphragm movement, and respiratory insufficiency. The aim was to compare the effect of Acapella device and breathing exercises with traditional chest physiotherapy program on forced expiratory volume/ forced vital capacity ratio after upper abdominal surgeries. Subjects and methods: Thirty patients underwent upper abdominal surgery were assigned randomly into two equal groups; their ages ranged from 20-50 years. The study group received breathing exercises, Acapella device and traditional chest physical therapy program (postural drainage. percussion, vibration, cough training and early ambulation). Control group received traditional chest physical therapy program (postural drainage. percussion, vibration, cough training and early ambulation). All groups received three sessions per week for four successful weeks. The data were collected before and after the same period of treatment for both groups. Evaluation procedures were carried out to measure forced expiratory volume/ forced vital capacity ratio (FEV1/FVC ratio) using electronic spirometer. Results: Post treatment results showed that there was a significant difference in FEV1/FVC ratio in both groups in favor of the study group. Percentage of improvement of FEV1/ FVC ratio in the study group was $10.17 \%$, while it was $2.96 \%$ in the control group. Conclusion: Acapella device and breathing exercises were more effective in improvement of FEV1/FVC \% than traditional chest physical therapy after upper abdominal surgeries.
\end{abstract}

Keywords: Acapella Device, Breathing Exercises, Forced Expiratory Volume/Forced Vital Capacity Ratio, Spirometer, Upper Abdominal Surgeries

\section{Introduction}

Patients undergoing upper abdominal and thoracic surgery have a decreased postoperative vital capacity (VC), which contributes to development of hypoxemia. Thus, the incidence rate of post operative pulmonary complications (PPC) is substantially higher for thoracic and upper abdominal surgery than for lower abdominal surgery. This can be explained by diaphragmatic dysfunction [1]. Impaired clearance of sputum results in a vicious cycle of colonization and infection of bronchi with pathogenic organisms, dilation of bronchi and further production of sputum [2].
Forced expiratory volume/ forced vital capacity ratio is the relationship between forced expiratory volume in one second (FEV1) and forced vital capacity (FVC) is expressed as FEV1/FVC ratio or FEV1 \% which is a simple screening test that will assist in the diagnosis of any respiratory impairment. FEV1 is the amount of exhaled air during the first second of FVC maneuver [3]. Pulmonary function tests are often performed using spirometry and based on sex, height, weight and age. When the patient performs the test, actual results will be compared with the predicted value expected of a person of gender, height, and age to see if he or she falls within the "normal" range, or has a restrictive or obstructive 
component based on the results [4].

Methods of sputum clearance have over time developed from standard physiotherapy to include; percussion, vibration and cough in varying postural drainage positions to active cycle of breathing techniques (ACBT), Autogenic drainage (AD), positive expiratory pressure devices (PEP) and oscillating positive expiratory pressure devices; the Flutter and then Acapella. These more recent techniques rely on the principles of getting air behind the secretions to move from distal to proximal along the muco-cilliary escalator. This theory is based on a necessity for shearing forces on the mucus within those airways to move the sputum in a proximal direction to aid expectoration [5].

The Acapella is a respiratory rehabilitation device designed to aid sputum clearance. When the patient exhales through this device, continuous and oscillatory pressure levels are produced. The adequate practical use of the Acapella is critically dependent on the characteristics of the produced pressure, which include the production of a mean pressure $>$ or $=10 \mathrm{~cm} \mathrm{H}_{2} \mathrm{O}$ and a matching of the oscillation frequency with the respiratory-system resonance frequency, and/or with the frequency of ciliary movement (approximately $13 \mathrm{~Hz}$ ) [6]. The ability of the Acapella to produce effective oscillations at low flows $(5 \mathrm{~L} / \mathrm{min})$ allows the use of oscillatory positive expiratory pressure devices (OPEP) with a broader spectrum of patients. Patients with low expiratory flow due to severe air flow obstruction, age, and/or size may now be included among those who are able to perform and perhaps benefit from OPEP. Because the Acapella is not gravity-dependent, it may be used while positioning the patient for postural drainage [7].

The aim of this study was to compare the effect of Acapella device and breathing exercises with traditional chest physiotherapy program on forced expiratory volume/forced vital capacity ratio after upper abdominal surgeries.

\section{Subjects, Materials and Methods}

This study was carried out on 30 adult patients of both sexes suffering from pulmonary complications and accumulation of secretions after upper abdominal surgery, their ages ranged from 20 to 50 years and selected from general surgery department at Om El Masryeen Hospital, Giza, Egypt during the period of February 2015 to August 2015. Patients were randomly assigned into two equal groups; Group A (Study group) and Group B (Control group). Group A received deep breathing exercises, Acapella device and traditional chest physical therapy program (postural drainage. percussion, vibration, cough training and early ambulation) and group B received traditional chest physical therapy program (Postural drainage, percussion, vibration, cough training and early ambulation).

Patients with the following conditions were excluded from the study; malignant disease, infection, active inflammation or sepsis. Patients spent more than 48 hours on mechanical ventilation, Instability of patient's medical condition, heavy smokers, alcohol drinking, patients with FEV1/FVC \% more than $80 \%$ and patients with any spirometry contra-indications (e.g Unstable cardiovascular status, pneumothorax, active hemoptysis and abdominal or cerebral aneurysms) were also excluded.

The work has been carried out in accordance with the ethics of committee for experiments at Faculty of Physical Therapy, Cairo University involving humans, and parents filled approval consent to share in the study.

The assessment approach was: Spirometer which is a computerized instrument that records: $\mathrm{FEV1/FVC} \mathrm{ratio} \mathrm{at}$ the beginning of the study (1st day post operative) and after four successive weeks.

The Acapella device (Smiths Medical Inc, Carlsbad, California, USA) was used by all patients in the study. Each patient was trained by the chest physiotherapist to complete three sets of the following cycle for each treatment session: 10 breaths (each inhaling to three quarters of the maximum inspiratory capacity then a 3 seconds breath hold followed by exhalation to functional residual capacity) followed by two to three forced expiratory techniques (huffs) or coughs. The total treatment 20-30 minutes 3 days/week for 4 weeks.

\section{Statistical Analysis}

Descriptive statistics and T- test for comparison of the mean age of both groups. Independent T-test was used for comparison of FEV1/ FVC ratio between both groups. Paired T-test was used for comparison between pre and post treatment mean values of FEV1/ FVC ratio in each group. The level of significance for all statistical tests was set at $\mathrm{p}<$ 0.05. All statistical tests were performed through the statistical package for social studies. (SPSS) version 19 for windows (IBM SPSS, Chicago, IL, USA).

\section{Results}

The statistical analysis for patient's socio-demographic data (age, sex) revealed a non significant difference between both groups.

Results revealed that; There was a significant difference in mean values of FEV1/ FVC ratio between both groups pre treatment $(p=0.71)$. While there was a significant difference in FEV1/ FVC ratio in group A post treatment compared to that of group $\mathrm{B}(\mathrm{p}=0.02)$ as shown in table 1 and figure 1 .

Table 1. Pre and post treatment results of FEV1/ FVC ratio for both groups.

\begin{tabular}{lllll}
\hline & \multicolumn{2}{l}{ Pre treatment } & \multicolumn{2}{l}{ Post-treatment } \\
\cline { 2 - 5 } & Group A & Group B & Group A & Group B \\
\hline$\overline{\mathrm{X}}$ & 67.13 & 66.39 & 73.96 & 68.36 \\
$\mathrm{SD} \pm$ & \pm 5.23 & \pm 5.38 & \pm 6.48 & \pm 5.6 \\
t- value & 0.37 & & 2.48 & \\
p- value & 0.71 & & 0.02 & \\
Significance & Non significant & Significant \\
\hline
\end{tabular}




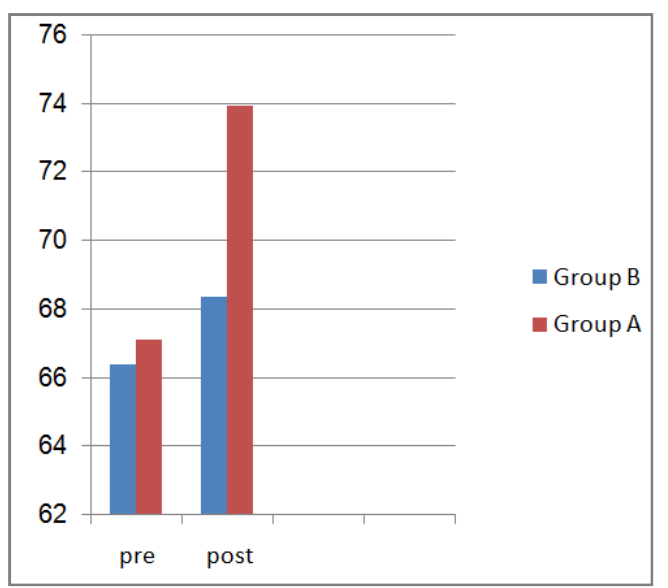

Figure 1. Pre and Post-treatment mean values of FEV1/ FVC \% for both groups.

Statistical analysis of results showed a significant improvement of FEV1/FVC \% in group A and in group B after 4 weeks of treatment as follows:

The mean \pm SD pre treatment of group A was $67.13 \pm$ $5.23 \%$ and that post treatment was $73.96 \pm 6.48 \%$. The mean difference was $-6.83 \%$ and the percent of improvement was $10.17 \%$. There was a significant increase in mean value of FEV1/FVC \% in group A post treatment compared with pre treatment $(\mathrm{p}=0.0001)$. While in group $\mathrm{B}$ the mean $\pm \mathrm{SD}$ of FEV1/FVC \% pre treatment was $66.39 \pm 5.38 \%$ and that post treatment was $68.36 \pm 5.6 \%$. The mean difference was $1.97 \%$ and the percent of improvement was $2.96 \%$. There was a significant difference in mean value of FEV1/FVC \% post treatment compared with pre treatment in group B (p $=0.0001)$ as shown in table 2 and figure 2 .

Table 2. Pre and post treatment results of FEV1/FVC \% for each group.

\begin{tabular}{lllll}
\hline & Group A & Group B \\
\cline { 2 - 5 } & Pre & Post & Pre & Post \\
\hline$\overline{\mathrm{X}}$ & 67.13 & 73.96 & 66.39 & 68.36 \\
$\mathrm{SD} \pm$ & \pm 5.23 & \pm 6.48 & \pm 5.38 & \pm 5.6 \\
$\mathrm{t}-$ value & -7.5 & & -12.72 & \\
p- value & 0.0001 & & 0.0001 & \\
Significance & Significant & & Significant & \\
\hline
\end{tabular}

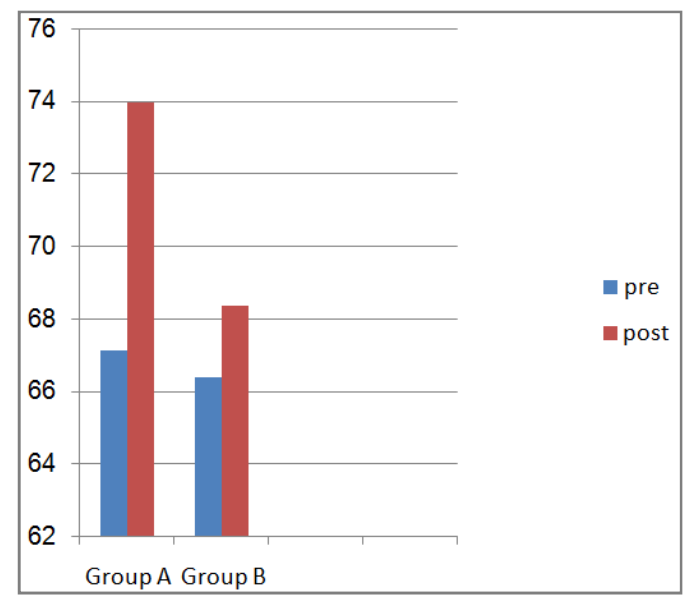

Figure 2. Mean values of pre and post treatment of FEV1/FVC \% for each group.

\section{Discussion}

The findings of this study indicated that there was a significant difference in FEV1/FVC \% after application of Acapella device with breathing exercises after upper abdominal surgeries. The statistical analysis revealed that the percentage of improvement of FEV1/FVC \% in the study group was $10.17 \%$ and that of the control group was $2.96 \%$. These results are consistent with authors who supported that Acapella device and breathing exercises is effective in improving $\mathrm{FEV} 1 / \mathrm{FVC} \%$ and reducing sputum after upper abdominal surgery as follows:

Pulmonary function tests: forced vital capacity (FVC), forced expiratory volume in the first second (FEV1), FEV1/FVC ratio, peak expiratory flow (PEF) and diaphragm excursion values between preoperative and postoperative (first, second) days were found to be higher in the exercise group when compared to control group performed on twenty sample sizes who underwent laparoscopic surgery were randomly divided as follows: 10 subjects performed diaphragmatic breathing exercise, (other therapies like bronchial hygiene therapy, Thoracic mobility exercise and mobilization) and 10 participated as a control group [8].

There were statistically significant differences between FlutterVRP1 and Shaker devices from Acapella, with their pressure amplitude values being higher than that of Acapella $\left(P \_\right.$.04). The authors concluded that the Flutter, VRP1 and Shaker devices had a similar performance to that of Acapella in many aspects, except for PEP [9]. It was found that there were significant difference in $\mathrm{FVC}$, FEV1, also $(\mathrm{PaO} 2)$ and carbon dioxide pressure ( $\mathrm{PaCO} 2)$ in group-A who received positive expiratory pressure breathing (PEPB) plus traditional physical therapy program (TPTP) than group-B who received TPTP only after 4 weeks [10].

Application of Acapella device for chest physiotherapy when compared with multimodality chest physiotherapy resulted in significant increase in the mean values of sputum amount, significant decrease in sputum viscosity, improved radiological signs of atelactasis, shorter duration of ventilator support, and less ICU stay. They concluded that Acapella device is a good representative to all conventional multimodality chest physiotherapy procedures with high success rate and can replace the exhausting, costy, and time consuming conventional procedures in such patients [11].

The OPEP devices have shown a significant increase in lung function parameters (FVC, FEV1 and forced expiratory flow) after more prolonged use in an outpatient setting [12]. The OPEP provide an improvement both in pulmonary function tests (FVC, FEV1) and quality of life. This treatment should be included among the principal options in chest physiotherapy [13].

Positive expiratory pressure technique provides a significantly greater improvement in the pulmonary function when compared to postural drainage with percussion. Other procedures are also utilized such as the Flutter device, autogenic drainage, the positive expiratory pressure technique, forced expiration techniques and intrapulmonary 
percussive ventilation [14].

In contrast, there were no significant differences in weight of sputum (during treatment plus $30 \mathrm{~min}$ after treatment), number of coughs, lung function [FEV1, FVC and peak expiratory flow (PEF)] and arterial oxygen pressure (SpO2). No significant differences were found between weight of sputum expectorated with ACBT treatment and weight of sputum expectorated with Acapella treatment - mean difference $0.54 \mathrm{~g}(95 \% \mathrm{CI}-0.39$ to 1.46$)$. A greater proportion of patients preferred Acapella. They concluded that Acapella is as effective method of airway clearance as active cycle of breathing techniques (ACBT) and may offer a user-friendly alternative to ACBT for patients with bronchiectasis [15].

Patients with bronchiectasis had no significant differences in sputum amount, FVC, FEV1, forced expiratory flow at $25-75 \%$ of $\mathrm{FVC}\left(\mathrm{FEF}_{25-75 \%}\right)$, FEV1/FVC \%, maximum inspiratory pressure (MIP) and maximum expiratory pressure (MEP). This study investigated the efficacy of regular chest physiotherapy using an oscillatory positive expiratory pressure device (Acapella device) group compared with no regular chest physiotherapy group. This is due to the underlying lung disorders and their old age as with increasing age, there is less airway reversibility, perhaps further limiting the opportunity for any benefit from chest physiotherapy on FVC or FEV1 [16].

There were no statistically significant differences among the airway clearance regimens of the active cycle of breathing techniques (ACBT), autogenic drainage (AD), positive expiratory pressure (PEP) and the oscillating PEP devices of the Flutter and RC when used over a period of one year, in the sitting position, by adults with cystic fibrosis. It is likely that to optimize adherence to treatment and consequently improvements in morbidity and mortality, the patient should be involved in the selection of his/her airway clearance regimen [17].

From the gained results it could be concluded that Acapella device and breathing exercises were more effective in improvement of $\mathrm{FEV} 1 / \mathrm{FVC} \%$ than traditional chest physical therapy after upper abdominal surgeries.

\section{References}

[1] Makhabah D, Martino F and Ambrosino N. Peri-operative physiotherapy. Multidisciplinary Respiratory Medicine; 2013. 8: 4-6.

[2] Barker A. Medical progress bronchiectasis. N Engl J Med; 2002. 346: 1383-1393.

[3] Dean $R$ and Robert $M$. Indications and initial settings for mechanical ventilation. Essentials of Mechanical ventilation. New York: McGraw- Hill; 2003. (2): 115-120.

[4] Veezhinathan $M$ and Ramakrishnan S. Detection of obstructive respiratory abnormality using flow-volume spirometry and radial basis function neural networks. J Med Syst; 2007. 31: 461-465.

[5] Lapin C. Airway physiology, autogenic drainage and active cycle of breathing. Respiratory Care; 2002.47 (7): 778-785.

[6] Alves Silva C, Santos J, Jansen J and De Melo P. Laboratory evaluation of the Acapella device: Pressure characteristics under different conditions and a software tool to optimize its practical use. Respir Care.; 2009, 54 (11): 1480-1487.

[7] Volsko T, Difiore J and Chatburn R. Performance comparison of two oscillating positive expiratory pressure devices: Acapella versus flutter. Respir Care; 2003. 48: 124-130.

[8] Augustine A, AlaparthiG, Anand R and Mahale A. Chest physiotherapy during immediate postoperative period among patients undergoing laparoscopic surgery - a randomized controlled pilot trail. International Journal of Biomedical and Advance Research; 2013, 4(2): 118-122.

[9] Dos Santos A, Guimarães R, Carvalho M and Gastaldi A. Mechanical Behaviors of Flutter VRP1, Shaker, and Acapella Devices. Respiratory care; 2013, 58(2): 298-304.

[10] Mohamed A, Taha M and Bahey El-Deen H. Effects of positive expiratory pressure breathing on functional outcomes in patients with chronic obstructive pulmonary disease after major abdominal surgeries: randomized controlled trial. International Journal of Advanced Research; 2014, (2)7: 784801.

[11] Abu-Rayan M, Afifi M, Othman M, El-Ganady A and Mikhael B. Evaluation of the single and combined roles of oscillating positive expiratory pressure device and conventional multimodality chest physiotherapy in mechanically ventilated COPD. Alexandria Journal of medicine; 2009, 45 (2): 354-364.

[12] Wolkove N, Kamel H, Rotaple M and Baltzan M. Use of a mucus clearance device enhances the bronchodilator response in patients with stable COPD. Chest; 2002, (121): 702-707.

[13] Nicolini A, CardiniF, Landucci N, Lanata S, Ferrari-Bravo M and Barlascini C. Effectiveness of treatment with highfrequency chest wall oscillation in patients with bronchiectasis. Pulmonary Medicine; 2013, 13 (21): 2-8.

[14] Lamari N, Martins A, Oliveira J, Marino L and Valerio N. Bronchiectasis and clearence physiotherapy: emphasis in postural drainage and percussion. Braz J Cardiovasc Surg; 2006, 21(2), 206-210.

[15] Patterson J, Bradley J, Hewitt O, Bradbury I, and Elborn J. Airway clearance in bronchiectasis: A randomized crossover trial of active cycle of breathing techniques versus Acapella. Respiration; 2005, 72: 239-242.

[16] Murray M, Pentland J and Hill A. A randomized crossover trial of chest physiotherapy in non-cystic fibrosis bronchiectasis. European Respiratory Journal; 2009, 34(5): 1086-1092.

[17] Pryor J, Tannenbaum E, Scott S, Burgess J, Cramer D, Gyi K and Hodson M. Beyond postural drainage and percussion: Airway clearance in people with cystic fibrosis. Journal of Cystic Fibrosis; 2010, 9 (3), 187-192. 